\title{
Potential Use of Multifunctional Nanoparticles for the Treatment of Cardiovascular Diseases
}

\author{
Clement Kleinstreuer ${ }^{1,2 *}$, Sriram Vasudevan Chari ${ }^{1}$, Shantanu Vachhani ${ }^{1}$ \\ 'Department of Mechanical and Aerospace Engineering, North Carolina State University, Raleigh, NC 27695, United States \\ 2Joint UNC-NCSU Department of Biomedical Engineering, Raleigh, NC 27695, United States
}

\section{Article Info}

\section{Article Notes}

Received: May 21, 2018

Accepted: June 20, 2018

\section{${ }^{*}$ Correspondence:}

Dr. Clement Kleinstreuer, Joint UNC-NCSU Department of Biomedical Engineering, Raleigh, NC 27695, United States; Email: ck@ncsu.edu

(c) 2018 Kleinstreuer $C$. This article is distributed under the terms of the Creative Commons Attribution 4.0 International License.

\section{Keywords}

Multifunctional nanoparticles

Direct drug delivery

Computer simulations

\section{Introduction}

The advent of multifunctional nanoparticles (NPs) as drugcarriers has spawned a host of innovative strategies in diagnostics, imaging and personalized medicine ${ }^{1}$. NPs are being used for the detection, visualization and treatment of different tumors as well as for various cardiovascular diseases.

The advantages of these multifunctional nanoparticles over conventional medicine can be summarized as follows ${ }^{2}$ :

- The small size of these nanoparticles (NPs) implies a large surface area-to-volume ratio. This allows for dosage reduction and improvement in toxicity profile of a nanodrug. The large surface area also increases the dissolution rate and solubility, which serves to improve the intracellular uptake of these drugs.

- The pharmaceutical carrier encapsulates the nanodrug; thus, protecting it from external agents.

- The small size of nanodrugs allows them to readily diffuse through cell-membranes; or, in case of brain tumors, cross the blood-brain-barrier (BBB) through different uptake mechanisms.

- A controlled release of nanodrugs enables site-specific provision of therapeutic cargo. A multitude of parameters can be activated to ensure controlled release of a drug, such as temperature, $\mathrm{pH}$-level, enzyme activity, magnetic field, etc.

Nevertheless, for the NPs to have a chance to, say, attack cancer cells, they have to be very close to the predetermined site to be successful. Once at the target site, either passive or active targeting may result in a desired treatment efficacy. Alternative methodologies that bring the NPs from the release point to a diseased area include the use of external magnets to steer the magnetic NPs to the target as long as it is located near/at the body surface. A more generally applicable technique is direct drug-delivery from the injection point to the predetermined site via optimal positioning of a smart microcatheter ${ }^{3}$.

Figure 1 depicts a composite graph showing schematically different components of multifunctional NPs.

Such NPs can be designed with a custom tumor-targeting ligand, imaging agent, and biological/synthetic therapeutics, 


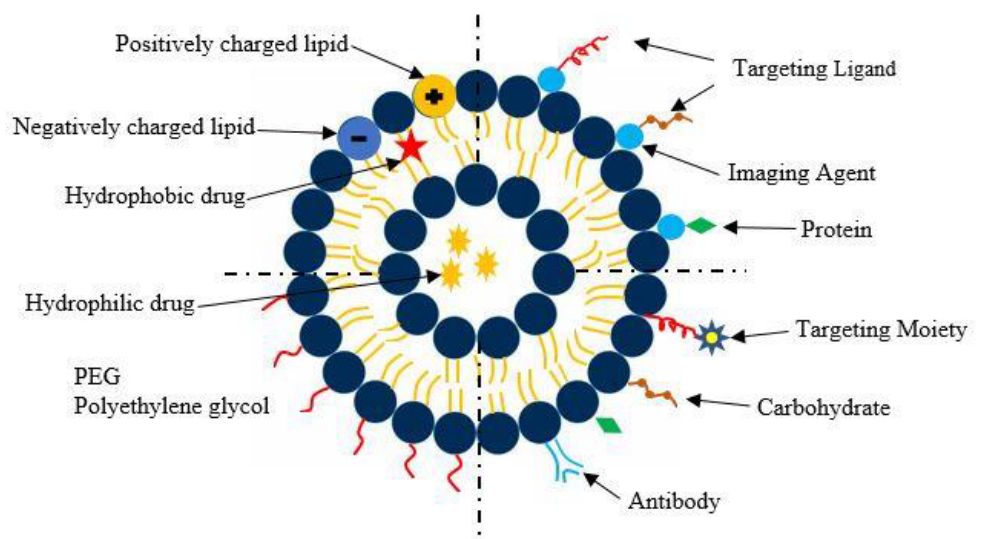

Figure 1: Representation of different liposomal drug carriers

all encapsulated within a drug-delivery vehicle ${ }^{4}$. The top left region shows a conventional liposomal drug delivery system. These liposomes consist of a lipid bilayer composed of positive ions, negative ions and neutrally charged particles. It has an aqueous solution surrounded by a hydrophobic membrane. The bottom left quadrant shows a liposome where the lipid layer is coated with PEG. PEGylation of liposomes is an effective strategy to enhance the delivery of therapeutic biomolecules such as proteins and carbohydrates ${ }^{5}$. The PEGylated biomolecules have increased stability and circulation times compared to their non - PEGylated counterparts which reduces both the frequency and dosage of administration of the drug. The bottom right quadrant shows ligands attached to the liposome. The ligands enable binding of the drug delivery carrier to the appropriate target site. The top left quadrant depicts a theranostic liposome where the liposome is directly connected to a functionalized imaging agent, a targeting moiety and a therapeutic drug, all encapsulated in a single system.

\section{Nano-medicines in Clinical Development}

Although numerous scientific articles on multifunctional nanoparticles are being published every year, the number of nanodrugs currently available for clinical use is only a tenth of what is reported in scientific studies ${ }^{2}$. A major reason for that is the commercialization costs involved in developing and marketing a nanodrug. The entire process, right from the inception of the drug to its market availability, can take anywhere between 10 to 15 years and costs upwards of $\$ 1$ billion $^{2}$. Therefore, a host of factors must be considered before commercializing a novel nanodrug.

As of October 2017, 56 clinical trials including the terms "nano" were listed as "Recruiting" or "Active" in ClinicalTrals.gov" ${ }^{6}$. The same search in April 2018 yielded 34 results, showing that the number of active trials declined when compared to what it was in 2017 . However, a closer look at the data will show that 44 clinical trials were declared "Completed" because they were not included in the earlier search. The number of nanodrugs that have received investigational new drug (IND) status has steadily increased since 2007, with the number peaking in the years from $2013-2015^{6}$. Again, a preliminary search with keywords "cardiovascular" and "nano" yielded two results, one of which was listed as "Recruiting" and the other as "Completed". So, it can be postulated that most of research in the field of multifunctional nanodrugs is focusing on tackling different types of cancerous tumors.

\section{Cardiovascular Applications}

Nanodrug research to cure cardiovascular diseases (CVD) is gaining traction and multifunctional NPs are used in the detection and treatment of CVD. Cardiovascular diseases are the leading cause of increasing mortality rates in the US7. Hypertension is often attributed as the key factor for the onset of cardiovascular diseases like myocardial infarction (MI), thrombosis, atherosclerosis, and restenosis.

Of all the cardiovascular diseases, atherosclerosis has received the most attention. Atherosclerosis (Figure 2) is the thickening of the arterial vessel wall due to plaque formation. The propagation of the lesions in atherosclerosis results in the formation of new blood vessels within the arterial walls like the growth of cancerous tumors. Vascular-targeted nano-carriers are increasingly used for the treatment of cardiovascular diseases. They allow for targeted delivery and increased efficacy of these nanodrugs. Nanotechnology can enable controlled delivery of active drugs, encapsulated in carriers, straight to the target site for the dissolution of atherosclerotic plaques that accumulate in the walls of the coronary arteries.

Restenosis, which is the recurrence of stenosis, usually pertains to an artery or other large blood vessel that has become narrowed, which results in reduced blood flow but higher wall-shear stresses. Drugs that can be used to combat restenosis can be classified into four groups: antiinflammatory, anti-thrombogenic, antiproliferative, and immunosuppressive ${ }^{8}$. 


\section{ATHEROSCLEROSIS}

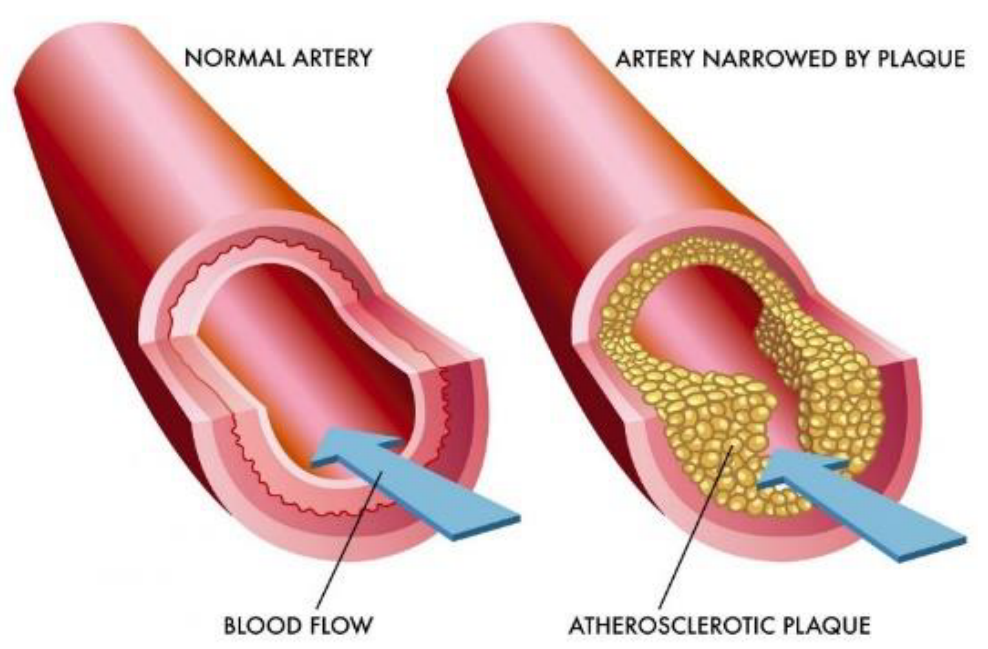

Figure 2: Atherosclerosis

https://universityhealthnews.com/daily/heart-health/recognize-atherosclerosis-symptoms-that-indicate-heart-disease/

Some of the important drugs tested to combat restenosis in vivo/clinical studies are reported in Table $1^{8}$.

Table 1: Drugs used to combat Restenosis in animals

\begin{tabular}{|l|l|l|}
\hline Drug & Animal & Region of Treatment \\
\hline Paclitaxel & Pig & Coronary artery \\
\hline $\begin{array}{l}\text { Sirolimus } \\
\text { (rapamycin) }\end{array}$ & Pig & Porcine aortic endothelial cells \\
\hline TRM - 484 & Rabbit & \\
\hline $\begin{array}{l}\text { Adenoviral vectors } \\
\text { containing human } \\
\text { inducible nitric oxide } \\
\text { synthase }\end{array}$ & Rat & Carotid \\
\hline
\end{tabular}

\section{DNA-enabled Drug Delivery Systems}

Recently many researchers have focused their attention on DNA nanostructures, possessing high biocompatibility, as potential drug-delivery vehicles. They are less prone to degradation when compared to synthetic NPs. This helps quell one of the major arguments against the use of NPs in drug delivery, which is the toxic nature of the NPs and their consequent harmful effects on the human body. Kumar et al. (2016) ${ }^{9}$ discussed the properties of DNA nanostructures and their use as potential nanodrug carriers for drug delivery to target cancerous cells. The concept of 'scaffolded DNA origami' ${ }^{10}$ is discussed which involves the folding of a long strand of DNA into a desired shape. This enables dense packaging of strands and controllable shapes which can be used to construct multifunctional drug carriers.

De Vries et al. (2018) ${ }^{11}$, demonstrated the efficacy of multifunctional NPs using DNA nanotechnology for targeted drug delivery in the treatment of ophthalmic diseases. The residence time of conventional eye drops is very short. Most of the droplets get cleared away by the eye-lid movement. This necessitates a high drug dosage. To overcome these shortcomings, they developed non - toxic, lipid modified DNA strands to form multifunctional NPs that adhere to the surface of the eye for an extended period. This has been proven in several ex - vivo experiments on human and porcine corneal rims and in an in - vivo animal model $^{11}$.

Li et al. (2018) $)^{12}$ devised a 'DNA walker' capable of cartwheeling rapidly along a predefined path. This unusual method of locomotion, i.e. moving from one foothold to another in a non - traditional manner but remaining secured to one foothold at all times, makes it faster than any device previously developed. These nano - walkers can also be made to carry cargo in the form of nanodrugs. The dynamical DNA nanodrug carriers allow for faster and more accurate delivery of drugs to the target site which may prove to be the difference between success and failure of a particular form of treatment.

A novel approach for drug delivery by joining drug molecules and polymer substrate by covalent bonds was described by Zhang et al. (2016) ${ }^{13}$ where doxorubicin was conjugated with a polymer consisting of folic acid. You et al $(2017)^{14}$ developed a drug with a polyethylene glycol and poly $\epsilon$-caprolactone linked copolymer with lecithin as the biodegradable material to transport cisplatin for breast cancer cell targeting. Similarly, for lung cancer, a cisplatin delivery vehicle consisting of organic block copolymer was the outcome of the study conducted by Wan et al. $(2018)^{15}$.

\section{Computer Simulations}

Blood is $45-50 \%$ composed of red blood cells (RBCs), 
i.e. the hematocrit, white blood cells (WBCs) and platelets - all embedded in the fluid plasma. With the advancement of computer technology, Computational Fluid-Particle Dynamics (CF-PD) simulations offer an encouraging option in tackling biomedical phenomena related to heterogeneity of blood flow and drug delivery.

\section{Direct Nanodrug Delivery for Tumor Targeting}

Targeted drug release systems target specific cancer cells, thereby minimizing side effects. This procedure takes advantage of the Enhanced Permeability and Retention (EPR) effect in the tumor vasculature, which allows for transport of the drug across porous membranes ${ }^{16}$. The hallmarks of EPR are a leaky vasculature and minimal lymphatic drainage. Blood vessels supplying tumor tissues have larger pore sizes compared to those in healthy tissue. This results in preferential accumulation of nanodrugs in the tumorous tissue by diffusion which increases treatment efficacy with reduced toxicity. Basciano et al. ${ }^{17}$ employed a one-way coupled Eulerian-Lagrangian model to study microsphere transport in the hepatic artery system (Figure 3) under steady state conditions. They demonstrated the generation of a particle release map (PRM) for optimal micro-catheter placement.

Most recently, $\mathrm{Xu} \&$ Kleinstreuer ${ }^{18}$ studied nanodrug delivery in the human hepatic artery system, focusing on the influence of RBCs on the nanodrug stream. The results provide a unique physical insight into the methodology employed to specifically target tumor-feeding capillaries. In this study, Lagrangian particle tracking was employed in conjunction with an optimal release location algorithm for a six-level vascular tree. With RBC deformation and inter-particle collision, facilitating drug delivery to target sites needs further research to properly quantify the effects of RBCs on nanodrug dispersion in micro-vessels. Experimental studies ${ }^{19}$ help to quantify the spreading of RBCs in blood vessels. So, the next step would be to incorporate these transport phenomena into a more advanced CF-PD model to capture the physics of blood flow and nanodrug delivery in a microenvironment.

\section{Arterial drug delivery}

The most effective and widely used treatment for cardiovascular diseases like ischemic heart disease and myocardial infarction (MI) involves heart transplants. However, due to the advancement of multifunctional nanoparticles, an alternative mode of treatment has gained attention. It has been noted that certain vasculature connected to the heart become more porous after $\mathrm{MI}^{20}$. This porous vasculature, like in the case of tumor-capillaries can utilize the EPR effect, allowing nanodrug transport for cell repair ${ }^{21}$. Vascular endothelial growth factor (VEGF) induces breakdown of the endothelial lining resulting in leaky vasculature ${ }^{20}$. In case of cancerous tumors, drug delivery systems (DDS) are highly dependent on the tumor vasculature. Excessive fluid accumulation in the tissue is a characteristic of human cancers. Anti - VEGF agents have shown to normalize these blood vessels by limiting the perfusion of fluid in the tissue surrounding the tumor. It has been observed that in the event of an ischemic stroke, the vascular leakage increases which results in the onset of edema. Production of anti - VEGF agents in response to stroke can limit infarct size which inhibits vascular leakage. Sukhovershin et al. (2016) ${ }^{22}$ discuss the

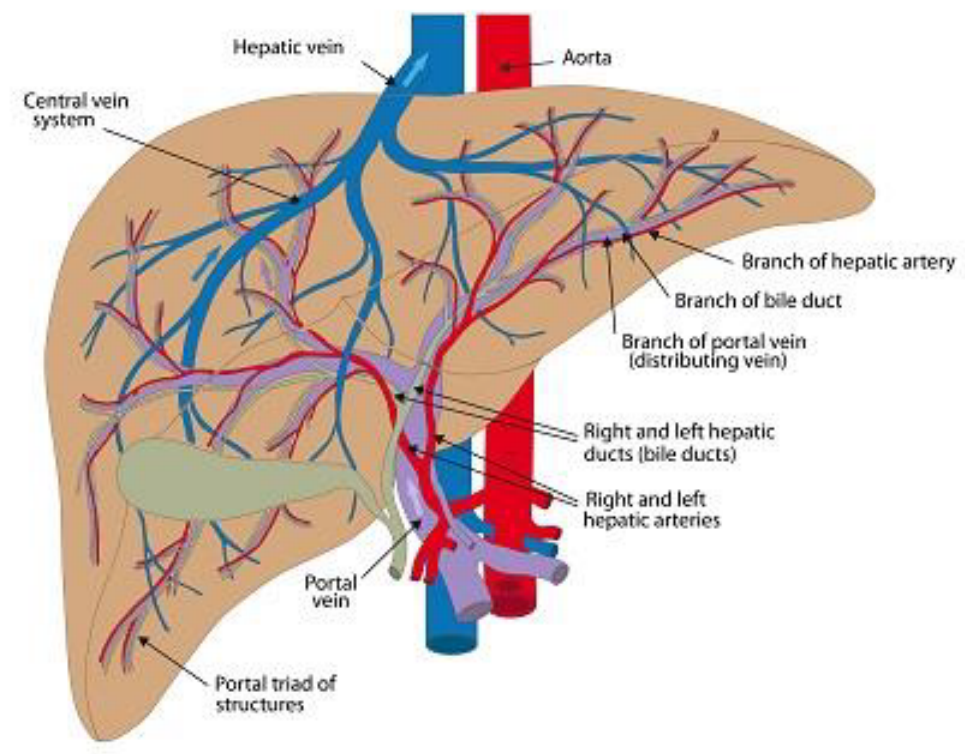

Figure 3: Human Hepatic Artery System

https://atlantavascularandveincenters.com/transarterial-chemoebolization-tace/ 
patho-biological factors contributing to atherosclerosis. Macrophages and smooth muscle cells (SMC) are mainly responsible for the growth of atherosclerotic plaque, making them natural targets for nano-therapies. Using nanotechnology to specifically target macrophage and SMC mediated inflammation without causing systemic toxicity is a promising strategy to suppress plaque progression ${ }^{23}$. Wong et al. $(2016)^{24}$ observed that under the influence of laminar shear stress, the endothelium releases nitric oxide and microRNAs that are resistant to atherosclerosis. It was found that these microRNAs suppress processes involved in atherosclerotic progression. They developed an E-selectin-targeted platform which serves as a docking site for the drug that enriches therapeutic microRNAs in the inflamed endothelium to inhibit formation of atherosclerotic plaques. Table 2 provides a brief summary of the many targeting methods discussed by Kelley et al. $(2016)^{25}$ in their review article which discusses strategies for the successful treatment of atherosclerosis using vascular targeted nano-carriers.

Kenjereš et al. ${ }^{26}$ modeled and simulated the phenomenon of atherosclerotic development (Figure 4). They employed magnetic drug targeting (MDT) to deliver drugs with magnetic cores to the area of plaque accumulation. Since this technique uses an external magnetic field for transport of the drugs, the efficiency of the drug transport increases significantly. Alternatively, for studying this problem the PRM-methodology ${ }^{18}$ could be employed to combat atheroclerosis via direct nanodrug delivery.

Forouzandehmehr et al. ${ }^{27}$ conducted an extensive study on margination and adhesion of micro/nano particles as a means to enhance the efficiency of drug delivery process for atherosclerotic plaque. The study aimed at quantifying the drug deposition/interaction at the raised patch or region, resulting from the deposition of fats. Modeling of these particles was done by incorporating drag and Brownian forces, while inter-particle interaction was done by using the Lennard-Jones potential for nanoparticles. Furthermore, the effect of particle shape and size on drug interaction with the plaque was studied, and it was shown that as the size increases the interaction with the plaque increases. The results of this study are important in order to design, both an effective drug and delivery method to mitigate various cardiovascular problems caused by atherosclerosis.

One of the major coronary artery ailments today is thrombosis. Drug-eluting stents (DES) are commonly used to mitigate this problem. Multiple stents are employed when thrombosis occurs at various points in an artery. Such a procedure may lead to overlapping stents, a phenomenon

Table 2: Summary of targeting schemes

\begin{tabular}{|c|c|c|c|}
\hline Method & Type & Advantages & Disadvantages \\
\hline Enhanced vascular permeability & Passive & No targeting ligands required & Non - specific accumulation \\
\hline Magnetic NPs & Pseudo - passive & No targeting ligands required & NPs must be magnetic \\
\hline Shear induced targeting & Passive & $\begin{array}{l}\text { Takes advantage of high laminar shear rates } \\
\text { in the vicinity of atherosclerotic plaques }\end{array}$ & Vessel must be highly occluded \\
\hline Multi - targeted nano-carriers & Active & $\begin{array}{l}\text { Specific tissues can be targeted using } \\
\text { multiple receptors }\end{array}$ & $\begin{array}{l}\text { Tend to be bigger and occupy more space } \\
\text { which can limit their effectiveness }\end{array}$ \\
\hline Collagen & Active & Targets injured epithelial lining & $\begin{array}{l}\text { The method is effective only if the } \\
\text { epithelium is injured }\end{array}$ \\
\hline
\end{tabular}

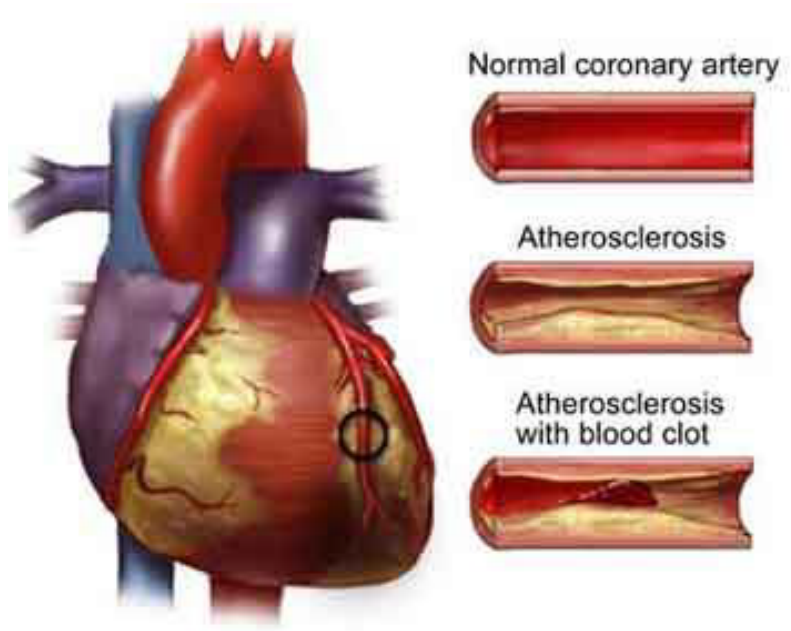

Figure 4: Formation of Blood Clot by Atherosclerosis 


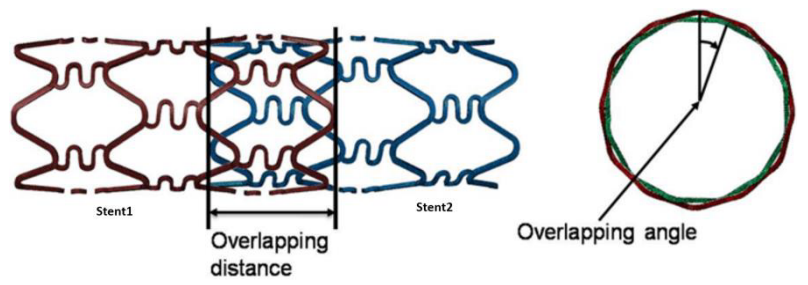

Figure 5: Overlapping Stents

http://medicaldevices.asmedigitalcollection. asme.org/article. aspx?articleid=2618343

investigated by Rikhtegar et al. ${ }^{28}$. They employed a computational model to study the flow and mass transfer based on an ex-vivo tomography of the vessels. This study presents useful results to aid in designing better and more efficient DES systems that optimize drug delivery. Future work would require incorporating transient effects of drug release and distribution along the arterial wall with drug removal via body metabolism.

\section{Conclusions}

Multifunctional nanoparticles have the potential for an expanded use in visualization, diagnostics and treatment of a variety of diseases. This mini-review discusses biological and synthetic nanoparticles and their applications to combat cardiovascular ailments. Specifically, different types of nanodrug targeting methods are outlined, numerous case studies are cited, and future work is discussed. A special emphasis is on computational fluidparticle simulations in support of direct nanodrug delivery for (liver) tumor targeting as well as for ways to mitigate cardiovascular complications. Simulation techniques have the advantage of being cost effective. If a template model is created, a technician just needs to input patient data like the hematocrit or a scanned copy of the occluded vasculature. This eliminates the need to have subject matter experts to run the gamut of activities before data is ready for analysis. of course, doctors have to analyze the data, but a host of intermediate activities can be expunged resulting in swifter data acquisition. However, we are still some way off from modeling these biological processes with a great deal of accuracy. Today, researchers are developing nanodrug carriers that mimic tissues and cells. The preliminary success of these bio - mimetic carriers suggests the need for a deeper understanding of the physiology of the tissue afflicted by CVD. Since funding for such ground-breaking research is contingent on data advocating the efficacy of these new technologies, further evidence in the form of clinical trials and long term follow up studies are required before these strategies can enter into mainstream practice. Clearly, inter-collaborative efforts are needed between clinicians, researchers and engineers, and with such sustained efforts the future of nanodrug delivery to combat cancers and CVD looks promising.

\section{Declarations of Interest}

None

\section{References}

1. $\mathrm{Xu} \mathrm{Z}$, Kleinstreuer C. Direct nanodrug delivery for tumor targeting subject to shear-augmented diffusion in blood flow. Med Biol Eng Comput. 2018: 1-10.

2. Fornaguera C, García-Celma MJ. Personalized nanomedicine: A revolution at the nanoscale. Journal of personalized medicine. 2017; 7(4): 12.

3. Kleinstreuer C, Feng Y, Childress E. Drug-targeting methodologies with applications: A review. World J Clin Cases. 2014; 2(12): 742-756. doi: 10.12998/wjcc.v2.i12.742 [doi].

4. Sercombe L, Veerati T, Moheimani F, et al. Advances and challenges of liposome assisted drug delivery. Frontiers in pharmacology. 2015; 6: 286.

5. Milla P, Dosio F, Cattel L. PEGylation of proteins and liposomes: A powerful and flexible strategy to improve the drug delivery. Curr Drug Metab. 2012; 13(1): 105-119.

6. Ventola CL. Progress in nanomedicine: Approved and investigational nanodrugs. Pharm Ther. 2017; 42(12): 742.

7. Martín Giménez VM, Kassuha DE, Manucha W. Nanomedicine applied to cardiovascular diseases: Latest developments. Therapeutic advances in cardiovascular disease. 2017; 11(4): 133-142.

8. Karimi M, Zare H, Bakhshian Nik A, et al. Nanotechnology in diagnosis and treatment of coronary artery disease. Nanomedicine. 2016; 11(5): 513-530.

9. Kumar V, Palazzolo S, Bayda S, et al. DNA nanotechnology for cancer therapy. Theranostics. 2016; 6(5): 710.

10. Castro CE, Kilchherr F, Kim D, et al. A primer to scaffolded DNA origami. Nature methods. 2011; 8(3): 221.

11. de Vries JW, Schnichels S, Hurst J, et al. DNA nanoparticles for ophthalmic drug delivery. Biomaterials. 2018; 157: 98-106.

12. Li J, Johnson-Buck A, Yang YR, et al. Exploring the speed limit of toehold exchange with a cartwheeling DNA acrobat. Nature nanotechnology. 2018: 1 .

13. Zhang Y, Teh $\mathrm{C}$, Li M, et al. Acid-responsive polymeric doxorubicin prodrug nanoparticles encapsulating a near-infrared dye for combined photothermal-chemotherapy. Chemistry of Materials. 2016; 28(19): 7039-7050.

14. You C, Wu H, Wang M, et al. Nearâ€ Infrared light and pH Dualâ€ Responsive targeted drug carrier based on Coreâ€ Crosslinked polyaniline nanoparticles for intracellular delivery of cisplatin. Chemistry-A European Journal. 2017; 23(22): 5352-5360.

15. Wan X, Min Y, Bludau H, et al. Drug combination synergy in worm-like polymeric micelles improves treatment outcome for small cell and non-small cell lung cancer. ACS nano. 2018; 12(3): 2426-2439. 
16. Matsumura Y, Maeda H. A new concept for macromolecular therapeutics in cancer chemotherapy: Mechanism of tumoritropic accumulation of proteins and the antitumor agent smancs. Cancer Res. 1986; 46(12 Part 1): 6387-6392.

17. Basciano CA, Kleinstreuer C, Kennedy AS, et al. Computer modeling of controlled microsphere release and targeting in a representative hepatic artery system. Ann Biomed Eng. 2010; 38(5): 1862-1879.

18. $\mathrm{Xu} \mathrm{Z}$, Kleinstreuer C. Direct nanodrug delivery for tumor targeting subject to shear-augmented diffusion in blood flow. Med Biol Eng Comput. 2018: 1-10.

19. Chuang C, Kikuchi K, Ueno H, et al. Collective spreading of red blood cells flowing in a microchannel. J Biomech. 2018.

20. Weis SM. Vascular permeability in cardiovascular disease and cancer. Curr Opin Hematol. 2008; 15(3): 243-249.

21. Ho YT, Poinard B, Kah JCY. Nanoparticle drug delivery systems and their use in cardiac tissue therapy. Nanomedicine. 2016; 11(6): 693714 .

22. Sukhovershin RA, Furman NET, Tasciotti E, et al. Local inhibition of macrophage and smooth muscle cell proliferation to suppress plaque progression. Methodist DeBakey cardiovascular journal. 2016; 12(3): 141.
23. Cooke JP, Atkins J. Nanotherapeutic solutions for cardiovascular disease. Methodist DeBakey cardiovascular journal. 2016; 12(3): 132.

24. Wong WT, Ma S, Tian XY, et al. Targeted delivery of shear stressinducible micrornas by nanoparticles to prevent vulnerable atherosclerotic lesions. Methodist DeBakey cardiovascular journal. 2016; 12(3): 152.

25. Kelley WJ, Safari H, Lopezâ€ Cazares G, et al. Vascularâ€ targeted nanocarriers: Design considerations and strategies for successful treatment of atherosclerosis and other vascular diseases. Wiley Interdisciplinary Reviews: Nanomedicine and Nanobiotechnology. 2016; 8(6): 909-926.

26. Kenjereš S. Modeling and simulation of multi-physics multi-scale transport phenomenain bio-medical applications. . 2014; 530(1): 012006.

27. Forouzandehmehr M, Shamloo A. Margination and adhesion of micro-and nanoparticles in the coronary circulation: A step towards optimised drug carrier design. Biomechanics and modeling in mechanobiology. 2018; 17(1): 205-221.

28. Rikhtegar F, Edelman ER, Olgac U, et al. Drug deposition in coronary arteries with overlapping drug-eluting stents. J Controlled Release. 2016; 238: 1-9. 